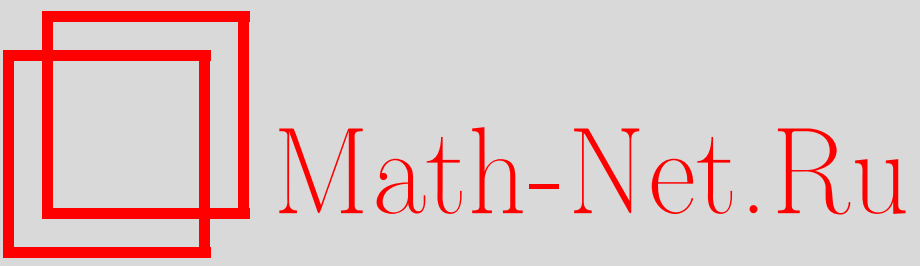

Д. П. Димитриченко, Об одном алгоритме обучения логических нейронных сетей, Итоги науки и техн. Сер. Соврем. мат. и ее прил. Темат. обз., 2019, том 166, 3-9

DOI: https://doi.org/10.36535/0233-6723-2019-166-3-9

Использование Общероссийского математического портала Math-Net.Ru подразумевает, что вы прочитали и согласны с пользовательским соглашением

http://www. mathnet.ru/rus/agreement

Параметры загрузки :

IP: 18.208 .226 .222

26 апреля 2023 г., 06:46:04 


\title{
ОБ ОДНОМ АЛГОРИТМЕ ОБУЧЕНИЯ ЛОГИЧЕСКИХ НЕЙРОННЫХ СЕТЕЙ
}

\author{
(c) 2019 г. Д. П. ДИМИТРИЧЕНКО
}

\begin{abstract}
АннотАция. В работе предложен алгоритм построения переменнозначных логических функций в случае добавления новых продукционных правил. Предложенный алгоритм служит основой метода конструктивного обучения логических нейронных сетей, построенных на основе переменнозначных предикатов.
\end{abstract}

Ключевъе слова: переменнозначный предикат, переменнозначная логическая функция, обучающая выборка, продукционное правило, логическая нейронная сеть, конструктивный алгоритм обучения.

\section{ON A CERTAIN LEARNING ALGORITHM FOR LOGICAL NEURAL NETWORKS}

\author{
(C) 2019 D. P. DIMITRICHENKO
}

\begin{abstract}
In this paper, we propose an algorithm for constructing variable-valued logical functions in the case of adding new production rules. The algorithm proposed serves as the basis for the method of constructive learning for logical neural networks based on variable-valued predicates.
\end{abstract}

Keywords and phrases: variable-valued predicate, variable-valued logical function, learning sample, production rule, logical neural network, constructive learning algorithm.

AMS Subject Classification: $68 \mathrm{~T} 27$

1. Введение. Нейронные сети хорошо зарекомендовали себя при решении задач распознавания образов и диагностики (см. [5]). В ряде случаев основой для построения эффективных процедур обучения являются конструктивные методы обучения нейронных сетей (см. [6]). В случаях, когда свойства анализируемых объектов задаются при помощи переменнозначных предикатов, а основные причинно-следственные связи фиксируются при помощи системы продукционных правил, то эффективным методом описания объектов и выявления на их основе скрытых закономерностей является метод построения переменнозначных логических функций (см. [4]). В [2,3] показана возможность представления переменнозначных логических функций при помощи логических нейронных сетей (см. [1]).

Особенность схемотехнического подхода (см. [1]) состоит в том, что логическая нейронная сеть рассматривается как сложное логическое высказывание, включающее в себя переменные и константы, связанные операциями конъюнкции и дизъюнкции. Для построения логической нейронной сети вводятся два типа нейронов: конъюнкторы и дизъюнкторы. Веса входных сигналов этих нейронов устанавливаются таким образом, чтобы при четких значениях входных сигналов

Работа выполнена при поддержке Российского фонда фундаментальных исследований (проект № 18-01-00050). 
на выходе этих нейронов вырабатывались бы совокупности результирующих сигналов, соответствующие значениям логических операций конъюнкции и дизъюнкции. Например, веса входов конъюнктора с $n$ входами устанавливаются равными $1 / n$. Таким образом, на выходе конъюнктора логическая единица будет генерироваться только тогда, когда все входы такого нейрона равны единице. Веса всех входов дизъюнктора устанавливаются достаточно близкими к единице, так чтобы для генерации логической единицы на выходе такого нейрона достаточно было бы присутствия хотя бы одной единицы на его входах.

Результат действия операции обобщенного отрицания фиксируется при формировании входного (рецепторного) слоя логической нейронной сети.

В этой связи особую актуальность приобретает задача построения алгоритма минимизации логических операций при добавлении новых продукционных правил в переменнозначную логическую функцию, содержащую ранее полученные продукционные правила, и, как следствие, адаптация полученного алгоритма для построения процедуры конструктивного обучения логических нейронных сетей, построенных на основе переменнозначных логических функций.

2. Общая постановка задачи. Приведем общую постановку задачи построения переменнозначной логической функции, содержащей описание свойств объектов при помощи переменнозначных предикатов.

Пусть $W=\left\{w_{1}, \ldots, w_{m}\right\}$ - заданное множество объектов, образующих обучающую выборку (OB) $W$. Описание объекта, принадлежащих ОВ $W$, представляет собой $n$-компонентный вектор, где $n$ - число признаков, используемых для характеристики анализируемого объекта $w$, причем $j$-я координата этого вектора равна значению $j$-го признака, $j=1, \ldots, n$. Число таких векторов равно $m$ по числу анализируемых объектов, входящих в ОВ $W$. Соответствие множества объектов и характеризующих их признаков представлено в следующей таблице:

\begin{tabular}{|c|c|c|c|c|}
\hline$x_{1}$ & $x_{2}$ & $\ldots$ & $x_{n}$ & $W$ \\
\hline$x_{1}\left(w_{1}\right)$ & $x_{2}\left(w_{1}\right)$ & $\ldots$ & $x_{n}\left(w_{1}\right)$ & $w_{1}$ \\
\hline$x_{1}\left(w_{2}\right)$ & $x_{2}\left(w_{2}\right)$ & $\ldots$ & $x_{n}\left(w_{2}\right)$ & $w_{2}$ \\
\hline$\ldots$ & $\ldots$ & $\ldots$ & $\ldots$ & $\ldots$ \\
\hline$x_{1}\left(w_{m}\right)$ & $x_{2}\left(w_{m}\right)$ & $\ldots$ & $x_{n}\left(w_{m}\right)$ & $w_{m}$ \\
\hline
\end{tabular}

Пусть $X_{j}=\left\{x_{1}\left(w_{j}\right), x_{2}\left(w_{j}\right), \ldots, x_{n}\left(w_{j}\right)\right\}$ - вектор качественных признаков, каждый элемент которого фиксированный признак характеризуемого объекта, и $W=\bigcup_{j=1}^{m} w_{j}$-множество характеризуемых объектов. Каждый соответствующий признак $x_{j}\left(w_{i}\right)$ в общем случае кодируется предикатом значности $k_{j}, j=1, \ldots, n$, т.е. переменнозначным предикатом:

$$
f(x)=\bigwedge_{j=1}^{m}\left(\bigwedge_{i=1}^{n} x_{i}\left(w_{j}\right) \rightarrow w_{j}\right)=\bigwedge_{j=1}^{m}\left(\bigvee_{i=1}^{n} \overline{x_{i}\left(w_{j}\right)} \vee w_{j}\right) .
$$

Указанный вид функции следует из известного логического тождества $a \rightarrow b=\bar{a} \vee b$, где $a-$ конъюнкция характеристик (признаков), определяющих объект, а $b$ - предикат, равный единице, когда $w_{j}$ становится равным соответствующему определяемому объекту. Такие предикаты будем называть объектными предикатами.

Поскольку эксперту часто приходится иметь дело с разнородным набором параметров (характеристик) исследуемых объектов, то переменнозначные предикаты как нельзя лучше подходят для наиболее адекватного кодирования свойств (признаков) анализируемых объектов, подбирая для каждого из них свою индивидуальную шкалу значений. Например, двузначный предикат, заданный на множестве значений «0» и «1» в состоянии кодировать наличие (или отсутствие) у анализируемого объекта некоторого свойства (характеристики). Трехзначный предикат, заданный на наборе свойств «холодно», «тепло», «жарко», способен легко кодировать общее представление об окружающей (или внутренней) диагностируемой температуре объекта.

Совокупность объектов и характеризующих их свойств (признаков), представленную в приведенной выше таблице легко преобразовать в логическую систему продукционных правил. Такая 
логическая система продукционных правил является основой для построения переменнозначной логической функции. Эта совокупность продукционных правил при помощи логических операций представима в следующем виде:

$$
\left\{\begin{array}{l}
\text { Конъюнкция признаков } 1 \rightarrow \text { объект } 1, \\
\text { Конъюнкция признаков } 2 \rightarrow \text { объект } 2, \\
\ldots \ldots \ldots \ldots \ldots \ldots \ldots \\
\text { Конъюнкция признаков } m \rightarrow \text { объект } m .
\end{array}\right.
$$

Совокупность из $m$ продукционных правил при помощи $m-1$ операции конъюнкции связывается в одно высказывание, порождающее переменнозначную логическую функцию $F(X, W)$. Эта функция с учетом предложенной в [4] операции обобщенного отрицания представима в виде конъюнктивной нормальная формы (КНФ). Последовательное раскрытие $m$ скобок приводит к построению переменнозначной логической функции $F(X, W)$ в виде дизъюнктивной нормальной формы (ДНФ), где $X-n$-компонентный вектор логических переменных (переменнозначных предикатов), кодирующий всю совокупность признаков объектов, принадлежащих ОВ $W$ : $X=\left\{x_{1}, x_{2}, \ldots, x_{n}\right\}$, а $W=\left\{w_{1}, \ldots, w_{m}\right\}$ - множество объектов, принадлежащих ОВ, мощности $m$.

В результате этого преобразования функция $F(X, W)$, принимает вид дизъюнкции, включающей в себя все возможные подклассы объектов, представляющих собой конъюнкцию только тех объектов и признаков по которым эти объекты совпадают, тем самым формируя соответствующие подклассы с различными степенями близости по числу признаков совпадения.

При нахождении значения логической функции $F(X, W)$ от заданного набора характеристик $X^{*}$ в качестве итогового результата формируется дизъюнкция тех подклассов объектов из множества $W$, значения переменных при которых совпадают со значениями переменных в заданном наборе запроса $X^{*}$. При этом, чем большему числу переменных из запроса $X^{*}$ соответствует объект $w^{*}$, принадлежащий ОВ $W$, тем большее число подклассов с его участием будет присутствовать в результирующем множестве $W^{*}: W^{*}=F\left(X^{*}, W\right)$. Отсюда следует, что для выявления наилучшего объекта, наиболее полно удовлетворяющего запросу $X^{*}$, достаточно к получившейся совокупности подклассов $W^{*}$ применить процедуру частотного анализа.

Теорема 1 (см. [3]). Всякая переменнозначная логическая функиия представима в виде логической нейронной сети, совокупность логических связей в которой взаимно однозначно определяется структурой продукиионных дизгюнктов и дизгюнктов свободных знаний.

Эта теорема позволяет сделать вывод о том, что оптимизация алгоритма преобразования переменнозначных логических функций позволит построить алгоритм конструктивного обучения логической нейронной сети, использующий особенности вычислений в алгебре логики при использовании переменнозначных предикатов.

3. Оценка верхней границы операций. Пусть в ранее сформированную переменнозначную логическую функцию $F(X, W)$, содержащую $m$ продукционных правил и состоящую из $l_{m-1}$ дизъюнктов, необходимо добавить новое правило вида $x \rightarrow w$.

С учетом операции обобщенного отрицания (см. [4]) получим:

$$
F(X, W)(x \rightarrow w)=F(X, W)(\bar{x} \vee w)=F(X, W)\left(\bar{x}_{1} \vee \bar{x}_{2} \vee \cdots \vee \bar{x}_{n} \vee w\right)
$$

Отсюда видно, что число требуемых умножений не превзойдет величины $l_{m-1} r$, где

$$
r=\left(\sum_{j=1}^{n}\left(k_{j}-1\right)\right)+1=\left(\sum_{j=1}^{n} k_{j}\right)-n+1
$$

Теперь в полученном множестве дизъюнктов (как новых, так и оставшихся без изменений) необходимо провести попарное сравнение дизъюнктов, при котором часть новообразованных дизъюнктов будет сокращена по закону поглощения. 
Поскольку верхняя граница оценки числа таких действий не превосходит квадратичной зависимости, то в итоге можем записать, что верхняя оценка действий, необходимых на $m$-м шаге добавлении нового правила $T_{m}$ не превзойдет величины $T_{m}: T_{m}=O\left(\left(l_{m-1} r\right)^{2}\right)$.

Заметим, что данная величина "-- это верхняя граница от новых полученных дизъюнктов, так как часть из них будет равна логическому нулю, поскольку умножение переменной самой на себя в различных состояниях (фазах) обнуляет результат этого произведения.

Очевидно, что в процессе реальных задач диагностики с более чем 100 объектов, каждый из которых определяется сотней характеристик, построение переменнозначной логической функции будет связано со значительным числом действий.

Требуется построить алгоритм изменения переменнозначной логической функции $F(X, W)$, характеризующийся меньшей вычислительной сложностью при добавлении новых продукционных правил.

\section{4. Исследование свойств переменнозначных функций.}

Определение 1. Множество характеристик, входящих в состав продукционных дизъюнктов будем называть связанными знаниями.

Предполагаем, как и ранее, что

(i) каждый объект характеризуется набором из $n$ различных характеристик;

(ii) добавляемые объекты (новые продукционные правила) будут представлены в дизъюнктивной форме (после применения к их характеристикам операции обобщенного отрицания).

Отсюда следует, что при добавлении в функцию $F(X, W)$ нового продукционного правила все содержащиеся в ней дизъюнкты последовательно будут умножатся либо на содержащиеся в них предикаты, либо на предикаты свободных знаний, либо на новый (добавляемый) объект.

Докажем следующие свойства.

Свойство 1. При умножении функции $F(X, W)$ на любой из предикатов связанных знаний результат его умножения на обгектный дизгюнкт будет сокращен по закону поглощения.

Доказательство. Действительно, объектный дизъюнкт $O$ содержит конъюнкцию всех ранее добавленных объектов множества ОВ $W$. Поскольку предикат $x$ принадлежит к связанным знаниям, то существует хотя бы один объект $w_{x}$, обладающий свойством $x$, и среди аксиоматических дизъюнктов содержится дизъюнкт вида $x \wedge w_{x}$. Тогда можем записать

$$
x \wedge\left(x w_{x} \vee O\right)=x \wedge w_{x} \vee x \wedge O=x \wedge w_{x}
$$

Свойство 1 доказано.

Свойство 2. Умножение переменнозначной логической функиии $F(X, W)$ на один из предикатов в результате оставляет без изменений все дизгюнкты, в которые он входит с учетом его состояния (фазы).

Доказательство. Доказательство для случая одной переменной является тривиальным, и его можно просто проиллюстрировать на конкретном примере.

Пусть множество объектов $W$ определено в рамках одного такого предиката $x$ значности $k_{x}=5$, что $X=\left\{x_{0}, x_{1}, \ldots, x_{4}\right\}$. Пусть при этом определена следующая простейшая система объектов $W=\left\{w_{0}, w_{1}, w_{2}\right\}$ со свойствами

$$
x_{0} \rightarrow w_{0}, \quad x_{1} \rightarrow w_{1}, \quad x_{2} \rightarrow w_{2} .
$$

Функция $F(X, W)$ будет иметь следующий вид:

$$
F(X, W)=\left(x_{0} \rightarrow w_{0}\right) \wedge\left(x_{1} \rightarrow w_{1}\right) \wedge\left(x_{2} \rightarrow w_{2}\right) .
$$


Воспользуемся указанной выше формулой преобразования импликации в дизъюнкцию с учетом операции обобщенного отрицания, применяемой к переменнозначным предикатам (см. [4]):

$$
\begin{aligned}
& F(X, W)=\left(x_{0} \rightarrow w_{0}\right) \wedge\left(x_{1} \rightarrow w_{1}\right) \wedge\left(x_{2} \rightarrow w_{2}\right)=\left(\bar{x}_{0} \vee w_{0}\right) \wedge\left(\bar{x}_{1} \vee w_{1}\right) \wedge\left(\bar{x}_{2} \vee w_{2}\right)= \\
& =\left(x_{1} \vee x_{2} \vee x_{3} \vee x_{4} \vee w_{0}\right) \wedge\left(x_{0} \vee x_{2} \vee x_{3} \vee x_{4} \vee w_{2}\right) \wedge\left(x_{0} \vee x_{1} \vee x_{3} \vee x_{4} \vee w_{3}\right)= \\
& =x_{0} \wedge w_{0} \vee x_{1} \wedge w_{1} \vee x_{2} \wedge w_{2} \vee x_{3} \vee x_{4} \vee w_{0} \wedge w_{1} \wedge w_{2} \text {. }
\end{aligned}
$$

При раскрытии скобок мы воспользовались тем правилом, что конъюнкция переменной $x$ в неравных (несовпадающих) состояниях (фазах) равна логическому нулю. Пусть данную логическую функцию необходимо пополнить новым продукционным правилом вида $x_{3} \rightarrow w_{3}$. Эта операция выполняется путем логического умножения текущей функции $F(X, W)$ на новое продукционное правило $x_{3} \rightarrow w_{3}$. В результате получим:

$$
\begin{aligned}
F(X, W) \wedge & \left(x_{3} \rightarrow w_{3}\right)= \\
& =\left(X_{0} \wedge w_{0} \vee x_{1} \wedge w_{1} \vee x_{2} \wedge w_{2} \vee x_{3} \vee x_{4} \vee w_{0} \wedge w_{1} \wedge w_{2}\right) \wedge\left(\bar{x}_{3} \vee w_{3}\right)= \\
& =\left(x_{0} \wedge w_{0} \vee x_{1} \wedge w_{1} \vee x_{2} \wedge w_{2} \vee x_{3} \vee x_{4} \vee w_{0} \wedge w_{1} \wedge w_{2}\right) \wedge\left(x_{0} \vee x_{1} \vee x_{2} \vee x_{4} \vee w_{3}\right) .
\end{aligned}
$$

Нетрудно заметить, что умножение первого выражения (функции $F(X, W)$ ) на переменную $x_{0}$ приведет к тому, что аксиома $x_{0} \wedge w_{0}$ останется без изменений, а остальные аксиомы будут обращены в нуль, так как результат перемножения переменной $x$ на саму себя при попарно различных фазах равен нулю. Дизъюнкт $x_{0} \wedge w_{0} \wedge w_{1} \wedge w_{2}$ будет сокращен по закону поглощения результирующей аксиомой $x_{0} \wedge w_{0}$.

Аналогичным образом эти рассуждения верны и для предиката $x$ в состояниях $x_{1}$ и $x_{2}$.

С учетом оставшихся без изменений первоначальных аксиом и свободных переменных все итоговые слагаемые, полученные при умножении функции $F(X, W)$ на объектный предикат $w_{3}$ будут также сокращены по закону поглощения. В результате этого получим:

$$
\begin{gathered}
\left(x_{0} \wedge w_{0} \vee x_{1} \wedge w_{1} \vee x_{2} \wedge w_{2} \vee x_{3} \vee x_{4} \vee w_{0} \wedge w_{1} \wedge w_{2}\right) \wedge\left(x_{0} \vee x_{1} \vee x_{2} \vee x_{4} \vee w_{3}\right)= \\
=x_{0} \wedge w_{0} \vee x_{0} \wedge w_{0} \wedge w_{1} \wedge w_{2} \vee x_{1} \wedge w_{1} \vee x_{1} \wedge w_{0} \wedge w_{1} \wedge w_{2} \vee x_{2} \wedge w_{2} \vee \\
\vee x_{2} \wedge w_{0} \wedge w_{1} \wedge w_{2} \vee x_{4} \vee x_{4} \wedge w_{0} \wedge w_{1} \wedge w_{2} \vee x_{0} \wedge w_{0} \wedge w_{3} \vee x_{1} \wedge w_{1} \wedge \\
\wedge w_{3} \vee x_{2} \wedge w_{2} \wedge w_{3} \vee x_{3} \wedge w_{3} \vee x_{4} \wedge w_{3} \vee w_{0} \wedge w_{1} \wedge w_{2} \wedge w_{3}= \\
=x_{0} \wedge w_{0} \vee x_{1} \wedge w_{1} \vee x_{2} \wedge w_{2} \vee x_{3} \wedge w_{3} \vee x_{4} \vee w_{0} \wedge w_{1} \wedge w_{2} \wedge w_{3} .
\end{gathered}
$$

Таким образом функция $F(X, W)$, включившая новое продукционное правило, примет следующий вид:

$$
F(X, W)=x_{0} \wedge w_{0} \vee x_{1} \wedge w_{1} \vee x_{2} \wedge w_{2} \vee x_{3} \wedge w_{3} \vee x_{4} \vee w_{0} \wedge w_{1} \wedge w_{2} \wedge w_{3}
$$

Мы видим, что изменились только два элемента: член, содержащий переменную $x_{3}$, по которой и характеризовался добавляемый объект $w_{3}$ и объектный дизъюнкт, зафиксировав новое множество объектов $W$. Теперь рассмотрим случай, когда объекты из множества $W$ характеризуются двумя предикатами $x$ и $y$. В общем случае каждый из них обладает значностью $k_{x}$ и $k_{y}$, принимая максимальное значение $m_{x}=k_{x}-1$ и $m_{y}=k_{y}-1$, соответственно. Пусть первоначально обучающая выборка $W$ состоит из двух объектов $W=\left\{w_{0}, w_{1}\right\}$ (простейшая система знаний):

$$
\begin{array}{|l|l|l|}
\hline x_{0} & y_{0} & w_{0} \\
\hline x_{1} & y_{1} & w_{1} \\
\hline
\end{array}
$$

Функция $F(X, W)$ представима в виде следующей конъюнкции продукционных правил:

$$
F(X, W)=\left(x_{0} \wedge y_{0} \rightarrow w_{0}\right) \wedge\left(x_{1} \wedge y_{1} \rightarrow w_{1}\right) .
$$


Приведя импликацию к дизъюнктивной форме и раскрыв скобки аналогично тому, как это сделано выше, получим:

$$
\begin{aligned}
& F(X, W)=x_{0} \wedge w_{0} \vee x_{1} \wedge w_{1} \vee y_{0} \wedge w_{0} \vee y_{1} \wedge w_{1} \vee x_{0} \wedge y_{1} \vee x_{1} \wedge y_{0} \vee x_{2} \vee \cdots \vee \\
& \vee x_{m_{x}} \vee y_{2} \vee \cdots \vee y_{m_{y}} \vee w_{0} \wedge w_{1} .
\end{aligned}
$$

Сгруппируем все содержащиеся в полученной функции дизъюнкты в $k_{x}+k_{y}$ группы по следующему принципу: в первую группу $g_{x_{0}}$ объединим дизъюнкты, содержащие переменную $x_{0}$ :

$$
\begin{aligned}
G_{x_{0}}= & x_{0} \wedge w_{0} \vee x_{0} \wedge y_{1}, \\
G_{x_{1}}= & x_{1} \wedge w_{1} \vee x_{1} \wedge y_{0}, \\
& \ldots \ldots \ldots \ldots \\
& G_{x_{m x}}=x_{m x} \\
G_{y_{0}}= & y_{0} \wedge w_{0} \vee y_{0} \wedge x_{1}, \\
G_{y_{1}}= & y_{1} \wedge w_{1} \vee y_{1} \wedge x_{0}, \\
& \ldots \ldots \ldots \ldots \\
& G_{y m y}=y_{m y} .
\end{aligned}
$$

Учтем, что, как было показано выше, объектный дизъюнкт, умноженный на любую из переменных, обязательно будет сокращен по закону поглощения, поэтому его достаточно один раз умножить на предикат добавляемого объекта и этот результат включить в результирующий вид функции $F(X, W)$. Умножение группы дизъюнктов $G_{x_{0}}$ на $x_{0}$ сохранит всю эту группу дизъюнктов в полном составе, а следовательно, последующие умножения членов этой группы на любые другие переменные приведет лишь к их поглощению первоначальными дизъюнктами группы, т.е. $G_{x_{0}} \wedge x_{0}=G_{x_{0}}$. Отсюда следует равенство

$$
G_{x_{0}} \wedge\left(x_{0} \vee a\right)=G_{x_{0}} \vee G_{x_{0}} \wedge a=G_{x_{0}},
$$

где $a$ - другой предикат, не равный $x_{0}$. Аналогичным образом подобные равенства доказываются и для всех остальных групп дизъюнктов. Рассуждая аналогично полученный результат без труда можно распространить на случай $n$ актуальных характеристик объектов. Свойство доказано.

5. Построение оптимального алгоритма. Пусть задана переменнозначная логическая функция $F(X, W)$, зависящая от $n$ предикатов $X=\left(x_{1}, \ldots, x_{n}\right)$, каждая из которых определяется значностью $k_{j}, j=1, \ldots, n$ и содержащая $m$ объектов из множества $W$. Требуется выполнить добавление нового продукционного правила вида $X^{*} \rightarrow w^{*}$ за минимальное число действий.

Опираясь на доказанные свойства, мы можем предложить следующую схему алгоритма преобразования переменозначной логической функции $F(X, W)$ при добавлении нового продукционного правила.

1. Привести добавляемое продукционное правило $X^{*} \rightarrow w^{*}$ к дизъюнктивной форме $\bar{X}^{*} \vee w^{*}$.

2. Из множества дизъюнктов функции $F(X, W)$ выбрать все дизъюнкты групп, соответствующие переменным дизъюнктивной формы $\bar{X}^{*}$ нового (добавляемого) правила.

3. Выбранные дизъюнкты поместить в итоговый результат.

4. Оставшиеся после предыдущего шага дизъюнкты функции $F(X, W)$ попарно перемножить с переменными дизъюнктивной формы нового продукционного правила.

5. При необходимости провести сокращения.

6. Полученные на предыдущем шаге дизъюнкты поместить в итоговый результат.

7. Построение переменнозначной логической функции $F(X, W)$, включающей новое продукционное правило, включающее описание объекта $w^{*}$, завершено.

Полученный алгоритм может быть положен в основу метода конструктивного обучения логических нейронных сетей, построенных на основе переменнозначных логических функций. 


\section{СПИСОК ЛИТЕРАТУРЫ}

1. Барский А. Б. Логические нейронные сети. - Интернет-университет информационных технологий, 2007.

2. Димитриченко Д. П. Использование нейронных сетей для повышения эффективности переменнозначных логических функций// Вестн. ИрГТУ. - 2015. - 10, № 105. - С. 12-16.

3. Димитриченко Д. П. Применение переменнозначных логических функций и нейронных сетей в системах принятия решений// Вестн. КРАУНЦ. Физ.-мат. науки. - 2016. - 4-1, № 16. - С. 93-100.

4. Лютикова Л. А. Моделирование и минимизация баз знаний в терминах многозначной логики предикатов/ препринт. - Нальчик, 2006.

5. Хайкин C. Нейронные сети: полный курс. - Москва: Издательский дом Вильямс, 2008.

6. Шибзухов 3. М. Конструктивные методы обучения нейронных сетей. - М.: Наука, 2006.

Димитриченко Дмитрий Петрович

Институт прикладной математики и автоматизации,

Кабардино-Балкарский научный центр Российской академии наук, Нальчик

E-mail: dimdp@rambler.ru 